\title{
Evolution of Interdiffused Gaussian-Shape Nanolayer in Au-Si(111) System at Ambient Condition
}

\author{
J.K. Bal ${ }^{a}$ and S. Hazra ${ }^{b}$ \\ Surface Physics Division, Saha Institute of Nuclear Physics, \\ 1/AF Bidhannagar, Kolkata 700064, India \\ a jayantak.bal@saha.ac.in, ${ }^{b}$ satyajit.hazra@saha.ac.in
}

\begin{abstract}
Keywords: Gaussian-shape nanolayer; Diffusion and interface formation; Surface passivation; X-
\end{abstract} ray reflectometry; Scanning tunneling microscopy.

\begin{abstract}
Evolution of interdiffused Gaussian-shape nanolayer of $\mathrm{Au}-\mathrm{Si}$, formed due to diffusion of $\mathrm{Au}$ into $\mathrm{Si}(111)$ substrate at ambient conditions, depends strongly on the $\mathrm{Si}$ surface pretreatment/passivation conditions. Negligible diffusion in the Au-OSi(111) sample, confirms the strong barrier action of the oxide-layer against diffusion, while large diffusion in the Au-HSi(111) sample compared to that in the Au-BrSi(111) sample suggests that the H-passivated $\mathrm{Si}(111)$ surface is more stable. This nature of the $\mathrm{Au}-\mathrm{Si}(111)$ system is qualitatively similar to that of the $\mathrm{Au}-$ $\mathrm{Si}(001)$ system but it differs quantitatively. The size, electronegativity and bond-energy of the passivating elements and the number of dangling bonds on the Si surface influence the instability of the Si surface. This instability, parameterized by growth-time of oxide layer alone, can be utilized to tune the amount of diffusion into the sub-surface Si region. The distribution of growth-time and fractional passivated area, which are related to the improper Si surface passivation, are against such control and needs perfection.
\end{abstract}

\section{Introduction}

The interface formed due to the deposition of metal atoms on the elemental semiconductor substrates plays a significant role in the fabrication of micro-electronic devices and interconnects. $\mathrm{Au}-\mathrm{Si}$ is one such interface, which has longstanding interest for device fabrications [1,2]. It is known that the presence of a native oxide layer at the interface strongly suppresses the interdiffusion behavior across a metal-semiconductor interface [3,4]. Oxide growth, at ambient conditions, could be hindered by passivation of surface dangling bonds [5,6]. However, the stability of the Si surface after passivation with different elements (such as $\mathrm{H}, \mathrm{Br}$, O, etc.) can be different depending upon their bond strength and relative electronegativity, which can strongly influence the interdiffusion of $\mathrm{Au}$ into $\mathrm{Si}$. Indeed, a strong dependence of diffusion on passivation has been observed for the $\mathrm{Au}-\mathrm{Si}(001)$ system [4], which suggests that a H-passivated $\mathrm{Si}(001)$ surface is stable for several days in air, while a Br-passivated $\mathrm{Si}(001)$ surface is not so stable and accordingly large diffusion of $\mathrm{Au}$ takes place into $\mathrm{H}$-passivated $\mathrm{Si}(001)$ compared to the Br-passivated one. On the other hand, in some work it have been shown that a Br-passivated $\mathrm{Si}(111)$ surface is more stable compared to a HF treated $\mathrm{Si}(111)$ surface $[7,8]$. The effects of passivation on $\mathrm{Si}(001)$ and $\mathrm{Si}(111)$ surfaces [7,9], can be different due to the difference in number of surface dangling bonds associated with each Si atom. The possibility to understand the interdiffusion and to form control interdiffused nanolayer, created renewed interest to study the $\mathrm{Au}-\mathrm{Si}$ system. In another previous study of ours [10], it has been observed surprisingly that atmospheric pressure plays the main role in anomalous wave-like diffusion or movement of $\mathrm{Au}$ atoms into native oxide free $\mathrm{Si}$ at room temperature. Such diffusion strongly depends on the thickness and coverage of the top Au layer. However, what exactly happens (both qualitatively and quantitatively) when the same pretreatment, as we carried out for $\mathrm{Si}(001)$ surface [4], is applied on $\mathrm{Si}(111)$ surface, needs to be looked out properly. The above comparative studies have been made by keeping in mind two main things; a) outside pressure 
which is here atmospheric pressure that acts on the Au film should be nearly same in each case, b) the $\mathrm{Au}$ film thickness and coverage that determines the freeness of individual $\mathrm{Au}$ atom approximately be the same in each case. To control the diffusion in nanolevel different parameters which are responsible for interdiffusion are to be well known.

Table 1. Parameters, such as the diffusion length $(L)$ of Au into Si(111), diffused amount at the time of deposition $\left(M_{0}\right)$, growth time $(\tau)$ of blocking layer; and the surface height variation $\left(h_{p v}\right)$, island size $\left(R_{D}\right)$, saturation surface roughness $\left(\sigma_{0}\right)$, correlation length $(\xi)$ and the scaling exponent $(\alpha)$, of the $\mathrm{Au}$ thin films on differently pretreated $\mathrm{Si}(111)$ substrates obtained from the analysis of the x-ray reflectivity data and STM images respectively. Suffixes $i$ and $f$ represent parameters corresponding to the initial and final time of measurements, respectively.

\begin{tabular}{|c|c|c|c|c|c|c|c|c|c|}
\hline Sample & $\begin{array}{l}L_{i} \\
{[\mathrm{~nm}]}\end{array}$ & $\begin{array}{l}L_{f} \\
{[\mathrm{~nm}]}\end{array}$ & $\begin{array}{l}M_{0} \\
{\left[\mathrm{e} / \AA^{2}\right]}\end{array}$ & $\begin{array}{l}\tau \\
{[\text { day }]}\end{array}$ & $\begin{array}{l}h_{p v} \\
{[\mathrm{~nm}]}\end{array}$ & $\begin{array}{l}R_{D} \\
\text { [nm] }\end{array}$ & $\begin{array}{l}\xi\left(\xi^{\prime}\right) \\
{[\mathrm{nm}]}\end{array}$ & $\begin{array}{l}\sigma_{0}\left(\sigma_{0}^{\prime}\right) \\
{[\mathrm{nm}]}\end{array}$ & $\alpha\left(\alpha^{\prime}\right)$ \\
\hline $\mathrm{Au}-\mathrm{BrSi}(111)$ & 3.6 & 4.6 & 18 & $2 \pm 0.3$ & $4.8 \pm 0.4$ & $17 \pm 4$ & 10 & 0.57 & 0.77 \\
\hline $\mathrm{Au}-\mathrm{HSi}(111)$ & 4.0 & 9.4 & 31 & $5 \pm 0.5$ & $7.8 \pm 0.5$ & $16 \pm 4$ & $10(28)$ & $0.65(0.79)$ & $0.82(0.73)$ \\
\hline
\end{tabular}

In this paper, our aim is exactly to address the qualitative and quantitative differences between the $\mathrm{Au}$ diffusion not only into differently passivated substrates but also into differently oriented substrates. For that, the time-evolution Au-Si interdiffused layer has been monitored using the XRR technique, after depositing Au into the Si(111) surface passivated differently. Analysis suggests that the interdiffusion behavior in the $\mathrm{Au}-\mathrm{Si}(111)$ system is qualitatively similar but quantitatively different to that in the Au-Si(001) system. A possible reason for such similarity has been discussed, taking into account different analyzed parameters, which can also provide quantitative difference. Effects of interdiffusion on the topography and on its in-plane correlation have been identified by scanning tunneling microscopy (STM).

\section{Experiment}

Three sets of samples, designated as $\mathrm{Au}-\mathrm{OSi}(111), \mathrm{Au}-\mathrm{HSi}(111)$ and $\mathrm{Au}-\mathrm{BrSi}(111)$, have been prepared by depositing $\mathrm{Au}$ on differently pretreated $\mathrm{Si}(111)$ substrates. Details of samples preparation are similar to previous work [4]. All samples have been characterized, mainly using Xray reflectivity (XRR) and scanning tunneling microscopy (STM) techniques.

\section{Results and discussion}

Evolution of electron density profile. Fig. 1a shows the time evolution XRR data along with the analysed curve for the two samples, where changes with time have been observed. Analysis of the data have been performed using the Parratt formalism [11] and considering three layers (top Au layer, intermediate layer and diffused layer) plus substrate. The analyzed electron density profile (EDP) is shown in Fig. 1b, while different parameters are listed in Table 1. For the top layer, the thickness is almost fixed, while the electron density $(\rho)$ and/or interfacial roughness varies with time which is evident from EDP. On the other hand, both the thickness (L) and the electron density of the interdiffused layer vary with time. The increase of the interdiffused layer with time is compensated by the decrease in the electron density of the top Au layer. The variation is, however, different for different samples. For the Au-OSi(111) sample (not shown here), interdiffusion is very small and almost unchanged with time, while for the Au-HSi(111) sample, the interdiffusion and its variation is appreciable. The effect is intermediate for the Au-BrSi(111) sample. The presence of an interdiffused nanolayer has been verified from SIMS measurements for the Au-HSi(111) sample and shown in the inset of Fig. 1b. A small-hump in the Au signal, indicated by the arrow, seems to 
be there, which is well evident from the Au-Si signal. The cross-sectional SEM image by simply breaking the sample and EDP of the sample after removing top Au layer using aqua regia (not shown here) also confirm the real existence of Gaussian-shape diffused layer.
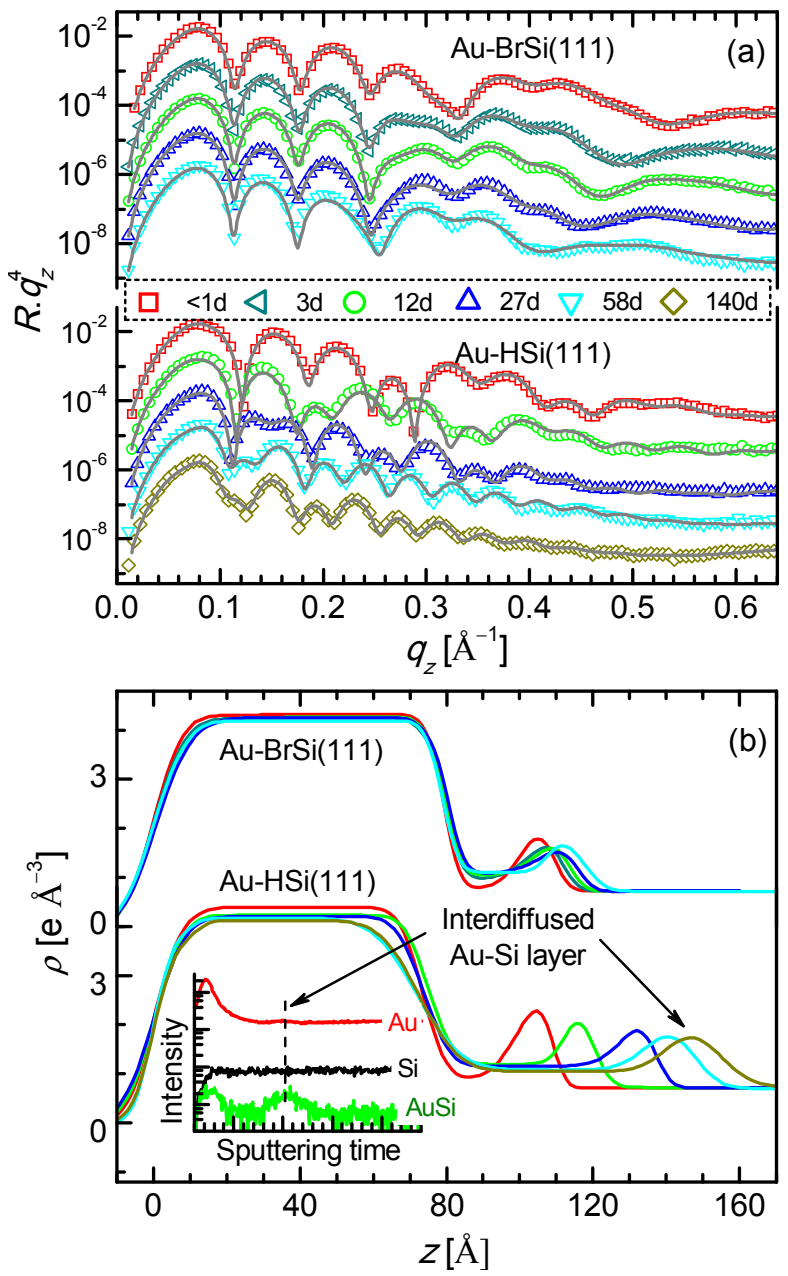

Figure 1: (Color online) (a) Time evolution normalized XRR data (different symbols and colors) and analyzed curves (solid line) of Au thin films on two differently pretreated Si(111) substrates in two panels. In each panel, curves are shifted vertically for clarity. (b) Corresponding analyzed EDP in different color for denoting time and in two panels for two samples. In legends, ' $d$ ' indicates the time in day. Inset: SIMS line profiles of $\mathrm{Au}, \mathrm{Si}$ and $\mathrm{Au}-\mathrm{Si}$ materials for $\mathrm{Au}-\mathrm{HSi}(111)$ sample after prolonged diffusion. Position of interdiffused Au-Si layer is indicated by the arrow.

Interdiffusion: Time dependence. The variation of diffused amount $(M)$ of Au with time (t) are shown in Fig. 2 for the Au-BrSi(111), Au-HSi(111) and Au-HSi(001), Au-BrSi(001) samples. The data for latter two samples has been taken from our previous work [4] and plotted to compare with the former samples. The strong deviation from the Fickian $t^{1 / 2}$ dependence [12] has been analyzed using a more generalized Fickian diffusion of the following form [4] where stretched exponential growth $[13,14]$ of a blocking (oxide) layer at the interface has been taken into account:

$$
M(t)=M_{0}+\Delta M^{\prime}\left[f \int_{0}^{t} e^{-\left(t^{\prime} / \tau\right)^{\beta}} t^{\prime 1 / 2} d t^{\prime}+(1-f) t^{1 / 2}\right] .
$$

The analyzed curves are shown in Fig. 2 and the corresponding parameters are listed in Table 1 . Analysis suggests that the blocking layer grows in the major portion $(f \approx 0.8)$ of the Si surface having distribution $(\beta \approx 0.7)$ of growth time $(\tau)$ for both the samples. While, the value of $\tau$ for the 
$\mathrm{Au}-\mathrm{BrSi}(111)$ sample is found less than that for the Au-HSi(111) sample indicating that the Si surface for the latter sample remains passivated for a longer time, for which large diffusion takes place. Values of $M_{0}$ suggest large diffusion during deposition in the Au-HSi(111) sample compared to that of the Au-BrSi(111) sample. This is likely to be related to the higher stability of the HSi(111) surface compared to that of the $\mathrm{BrSi}(111)$ surface and/or also to the smaller size of $\mathrm{H}$ atom compared to that of $\mathrm{Br}$ atom. Similar effect is also observed in the $\mathrm{Au}-\mathrm{Si}(001)$ system [4]. The value of $\tau$ for the Au-BrSi(111) sample is large (about one order) compared to that for the Au-BrSi(001) sample, while those for the Au-HSi(111) and Au-HSi(001) samples are almost same.

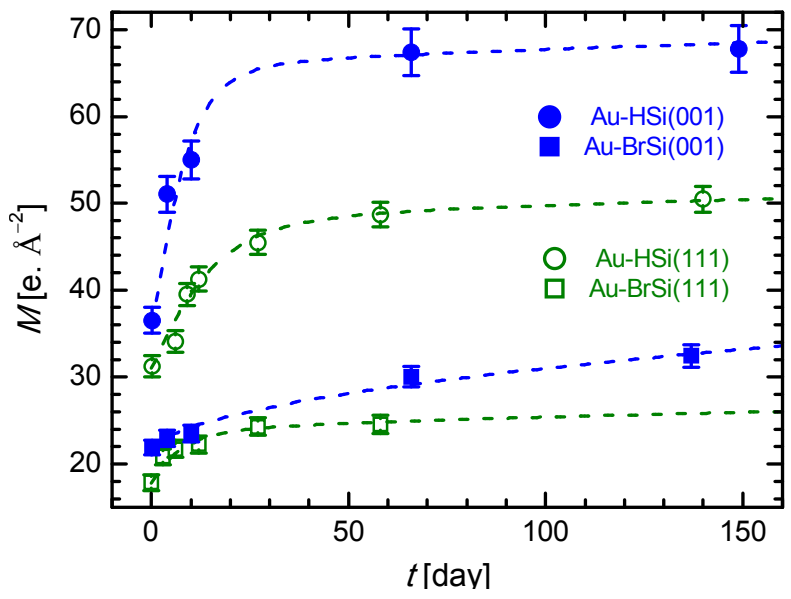

Figure 2: (Color online) The diffused amount of $\mathrm{Au}$ into the differently pretreated $\mathrm{Si}(111)$ and $\mathrm{Si}(001)$ substrates as a function of time. Dashed lines through the data are the analyzed curves using Eq. 1. An error bar has been incorporated for each data.

Topography and height-height correlation. Interfacial instability and interdiffusion can strongly modify the topography. Typical STM images, showing topography of the Au thin films after prolonged diffusion, are shown in Fig. 3. It can be noted that all films are composed of small islands of similar size. Additional modulation is observed above that, which is prominent for the AuHSi(111) sample. The height variation is large in this sample compared to $\mathrm{Au}-\mathrm{BrSi}(111)$ due to larger interdiffusion at the interface. Height-height (difference) correlation function $(g(r))$ obtained from the STM images have been analyzed considering kinetic rough surface [4,15-17]. Data and analyzed $g(r)$ for the two samples are shown in Fig. 3, while the parameters are listed in Table 1. Two distinct correlation lengths $\left(\xi\right.$ and $\xi$ ) with different saturation roughness $\left(\sigma_{0}\right.$ and $\left.\sigma_{0}\right)$ and scaling exponent ( $\alpha$ and $\alpha$ ) have been observed due to strong interdiffusion through the interface having a non-uniform blocking layer in the Au-HSi(111) sample. Large value of $\alpha$ along with small values of $\xi$ and $\sigma_{0}$ are associated with the normal in-plane diffusion dominated growth, while a comparatively small value of $\alpha^{\prime}$ and large values of $\xi^{\prime}$ and $\sigma_{0}^{\prime}$ are associated with the strong interdiffusion. However, in the present system, the values of $R_{D}$ and $\xi$ for all the samples are relatively small, while the value of $h_{p v}$ and $\sigma_{0}$ for $\mathrm{Au}-\mathrm{BrSi}(111)$ sample is relatively large. These can be attributed to the high-resolution of STM mode over AFM. Also, due to this high-resolution, two distinct correlation lengths ( $\xi$ and $\xi$ ) are observed in the Au-HSi(111) sample, which was however, smeared out in the Au-HSi(001) sample [4], to give one large value.

Mechanism behind instability and interdiffusion. The fact that the results of the present Au$\mathrm{Si}(111)$ system are qualitatively similar to those of the Au-Si(001) system, is something not expected and needs justification. Large diffusion, as obtained from both initial and final value of $M$, into the $\mathrm{Si}(001)$ sample compared to that into the $\mathrm{Si}(111)$ sample, especially through the $\mathrm{H}$ passivated surface, is related to the openness in their surface structure. As the density of atoms on the $\mathrm{Si}(111)$ surface is more $(2 / \sqrt{ } 3$ times) than that on the $\mathrm{Si}(001)$ surface [18], the open space in the 
latter is more which can provide larger diffusion. On the other hand, the number of dangling bonds associated with each surface atom are different in the two Si surfaces. Passivation of those dangling bonds with different materials and their stability not only depends on the relative electronegativity and bond-energy $(D)$ with $\mathrm{Si}$, but also on the atomic size $\left(R_{a}\right)$. Values of such parameters, obtained from the online sites $[19,20]$, are listed in Table 2. Small size $H$ atoms can passivate both the surfaces without facing any spatial problem, which provide comparatively large $\tau$ value. Big size $\mathrm{Br}$ atoms can face problem (steric hindrance) to saturate two dangling bonds of each $\mathrm{Si}$ atom on the $\mathrm{Si}(001)$ surface, as observed in low $\tau$ value. For the $\operatorname{Si}(111)$ surface, where one dangling bond is associated with each $\mathrm{Si}$ atom, such size is not a problem. If we consider the value of bond-energy and bond-length of $\mathrm{H}$ and $\mathrm{Br}$ with $\mathrm{Si}$ then it is obvious that dissociation of $\mathrm{H}$ is difficult compared to $\mathrm{Br}$ and also $\mathrm{Br}$ adsorption can induce a large reduction in the bond charge of Si-Si backbonds [5,21] which facilitates the desorption of $\mathrm{Br}$ unlike $\mathrm{H}$, where such effect is small. Above effects have been reflected on their corresponding $\tau$ values and helps in tuning interdiffusion.

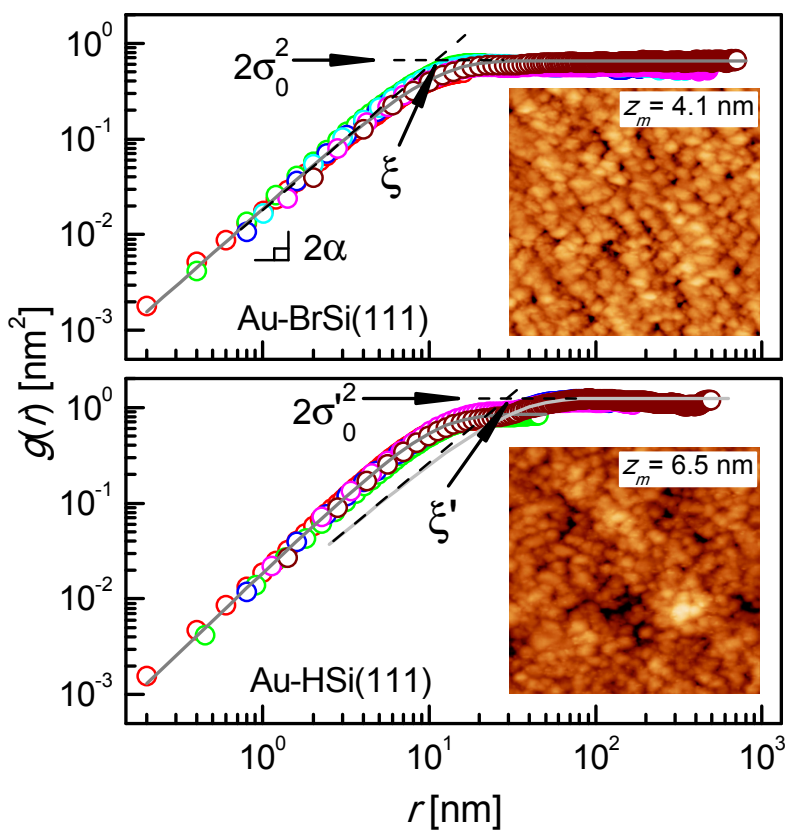

Figure 3: (Color Online) Height-height correlation along with the topography (of size $320 \times 320 \mathrm{~nm}^{2}$ ) in the inset for Au films on differently pretreated $\mathrm{Si}(111)$ substrates after prolonged diffusion in different panels. The symbols represent the data estimated from STM images of different scan sizes, while solid lines are the fits. Different parameters associated with the height-height correlation function are indicated by arrows for clarity. $z_{m}$ indicates maximum height variation.

The wave-like movement of diffused nanolayer as a whole is well understood by considering the atmospheric pressure as a main driving force where difference in pressure between atmospheric pressure and the inherent pressure inside the crystal structure acts on the interface [10]. Our result suggests that the inherent pressure is less by more than four orders of magnitude compared to the atmospheric pressure. Still it is not completely understood the formation of the Gaussian shape diffused nanolayer from the initial stage. We believe that the diffused layer takes such an interesting shape separated from the interface by a dip near the interface at the time of deposition when diffusion takes place between two different pressure mediums. In-situ X-ray reflectivity study by changing the external vacuum pressure is needed in order to understand completely. 
Table 2. Relevant parameters, such as the covalent atomic radii $\left(R_{a}\right)$, the electronegativity $(\delta)$ in Pauling scale, the bond-energy $(D)$, and the bond-length $\left(L_{B}\right)$ with Si for different elements, as obtained from online sites $[19,20]$.

\begin{tabular}{lllll}
\hline Element & $R_{a}$ & & $D$ & $L_{B}$ \\
\hline $\mathrm{Si}$ & {$[\AA]$} & $\delta$ & {$[\mathrm{kJ} / \mathrm{mol}]$} & {$[\AA]$} \\
$\mathrm{H}$ & 1.11 & 1.90 & 222 & 2.33 \\
$\mathrm{Br}$ & 0.37 & 2.20 & 318 & 1.48 \\
$\mathrm{O}$ & 1.14 & 2.96 & 310 & 2.15 \\
$\mathrm{~F}$ & 0.73 & 3.44 & 452 & 1.63 \\
& 0.71 & 3.98 & 565 & 1.60
\end{tabular}

\section{Conclusions}

The stability or instability of the $\mathrm{Au}-\mathrm{Si}(111)$ interface, at ambient conditions, have been studied from the interdiffusion and compared with those of the $\mathrm{Au}-\mathrm{Si}(001)$ interface. X-ray reflectivity measurements show different time-evolution for $\operatorname{Si}(111)$ surface pretreated differently. The evolution of the interdiffused amount has been fitted with more generalized Fickian-type diffusion, considering stretched exponential growth of a blocking layer at the interface, where the growth-time depends on the type of $\mathrm{Si}(111)$ surface passivation. In particular, the growth-time and hence the stability of the HF-treated surface is found large compared to that of the Br-treated one, similar to that observed for the Au-Si(001) system. The presence of the Gaussian shape Au-Si interdiffused layer is also evident from secondary ion mass spectrometry. Island-like topography, mapped by scanning tunneling microscopy and its height-height correlation, show different height-variation and correlation, consistent with the amount of interdiffusion and the growth of non-uniform blocking layer at the interface. Differences in the $\mathrm{Au}-\mathrm{XSi}(111)$ interfaces, for $\mathrm{X}=\mathrm{O}, \mathrm{Br}$ and $\mathrm{H}$; and its similarity with those of the $\mathrm{Au}-\mathrm{XSi}(001)$ interfaces have been well interpretated considering atomic size, electronegativity, etc. of element $\mathrm{X}$ and number of dangling bonds, open space, etc. of Si surfaces. Although the reason behind the movement of Gaussian-shape diffused nanolayer into $\mathrm{Si}$ is well understood, the reason behind such shape is very little understood and need further investigation.

\section{Acknowledgements}

The authors would like to thank Prof. Srinanda Kundu for her valuable help in gold deposition using magnetron sputtering unit and Mr. Biswajit Saha for the SIMS measurement.

\section{References}

[1] A. Cros, P. Muret: Mater. Sci. Rep. Vol. 8 (1992), p. 271.

[2] C.B. Collins, R.O. Carlson, C.J. Gallagher: Phys. Rev. Vol. 105 (1957), p. 1168.

[3] L.A. Baker, A.R. Laracuente and L. J. Whitman: Phys. Rev. B Vol. 71 (2005), p. 153302.

[4] J.K. Bal and S. Hazra: Phys. Rev. B Vol. 75 (2007), p. 205411.

[5] G.W. Trucks, K. Raghavachari, G.S. Higashi and Y.J. Chabal: Phys. Rev. Lett. Vol. 65 (1990), p. 504.

[6] J.K. Bal and S. Hazra: Phys. Rev. B Vol. 79 (2009), p. 155412.

[7] K. Sekar, P.V. Satyam, G. Kuri, D.P. Mahapatra and B.N. Dev: Nucl. Instrum. Meth. B Vol. 71 (1992), p. 308.

[8] C.H. Lee, Z.D. Lin, N.G. Shang, L.S. Liao et al.: Phys. Rev. B Vol. 62 (2000), p. 17134. 
[9] J.F. Chang, T.F. Young, Y.L. Yang, H.Y. Ueng, T.C. Chang: Mater. Chem. Phys. Vol. 83 (2004), p. 199.

[10] J.K. Bal and S. Hazra: Phys. Rev. B Vol. 79 (2009), p. 155405.

[11] L.G. Parratt: Phys. Rev. Vol. 95 (1954), p. 359.

[12] A. Fick: Phil. Mag. Vol. 10 (1855), p. 30.

[13] R. Kohlrausch: Pogg. Ann. Phys. Vol. 12 (1847), p. 393.

[14] G. Williams and D. C. Watts: Trans. Faraday Soc. Vol. 66 (1970), p. 80.

[15] J.K. Basu, S. Hazra and M.K. Sanyal: Phys. Rev. Lett. Vol. 82 (1999), p. 4675.

[16] S.K. Sinha, E. B. Sirota, S. Garoff and H. B. Stanley: Phys. Rev. B Vol. 38 (1988), p. 2297.

[17] A.-L. Barabási and H.E. Stanley: in Fractal Concepts in Surface Growth; University Press: Cambridge, 1995.

[18] A. Korkin, J.C. Greer, G. Bersuker, V.V. Karasiev and R.J. Bartlett: Phys. Rev. B Vol. 73 (2006), p. 165312.

[19] Periodic Table of Elements; http://www.dayah.com/periodic.

[20] Wired Chemist; http://www.wiredchemist.com.

[21] H. Aizawa, S. Tsuneyuki and T. Ogitsu: Surf. Sci. Vol. 438 (1999), p. 18. 